\title{
Ecoepidemiologia da paracoccidioidomicose: Uma revisão narrativa da literatura
}

\author{
Ecoepidemiology of paracoccidioidomycosis: A narrative review of the literature \\ Ecoepidemiología de la paracoccidioidomicosis: Uma revisión narrativa de la literatura
}

Recebido: 13/07/2021 | Revisado: 18/07/2021 | Aceito: 19/07/2021 | Publicado: 26/07/2021

\author{
Marcos Jessé Abrahão Silva \\ ORCID: https://orcid.org/0000-0003-2057-3474 \\ Universidade da Amazônia, Brasil \\ E-mail: jesseabrahao10@gmail.com \\ Eliete Costa da Cruz \\ ORCID: https://orcid.org/0000-0002-0180-5682 \\ Universidade da Amazônia, Brasil \\ E-mail: elietecruz.99@gmail.com \\ Geovanna Carla Bandeira Gama \\ ORCID: https://orcid.org/0000-0001-8695-3352 \\ Universidade da Amazônia, Brasil \\ E-mail: carlageovanna70@gmail.com \\ Andrei Santos Siqueira \\ ORCID: https://orcid.org/0000-0002-2397-7119 \\ Universidade Federal do Pará, Brasil \\ E-mail: andrei.siqueira@icb.ufpa.br
}

\begin{abstract}
Resumo
A paracoccidioidomicose é uma micose sistêmica prevalente na América Latina. É causada pelo Paracoccidioides brasiliensis e Paracoccidioides lutzii, que são fungos termodimórficos, a fase filamentosa está presente no solo de forma saprofítica. $\mathrm{O}$ objetivo deste trabalho é apresentar dados ecoepidemiológicos associando a fatores ecológicos e socioambientais da doença. Foi realizada uma revisão narrativa, por meio dos bancos de dados PubMed, Scielo, LILACS, MEDLINE e Google Scholar onde foram selecionados artigos, teses e dissertações que abordassem o tema entre 2000 e 2021. Foram encontrados 35 artigos, 2 dissertações, 2 teses e 1 monografia com foco de pesquisa no tema de interesse. Foi descrita a existência de outros hospedeiros, além do homem, principalmente tatus da espécie Dazypus novemcinctus auxiliando a analisar a situação de territorialidade, e delimitar as características do ecossistema do fungo. As mudanças socioambientais também influenciam na incidência da doença. Há uma forte associação no padrão epidemiológico e o ecossistema do Paracoccidioides, por essa análise é possível prever possíveis surtos e controlar a doença.
\end{abstract}

Palavras-chave: Ecoepidemiologia; Epidemiologia; Ecologia; Paracoccidioidomicose.

\begin{abstract}
Paracoccidioidomycosis is a systemic mycosis prevalent in Latin America, it is caused by Paracoccidioides brasiliensis and Paracoccidioides lutzii, which are thermodimorphic fungi, the filamentous phase is present in the soil in a saprophytic form. The objective of this work is to search presents ecoepidemiological data associating ecological and socio-environmental factors with the disease. The narrative review was carried out using the PubMed, Scielo, LILACS, MEDLINE and Google Scholar databases, where articles, theses and dissertations that addressed the topic between 2000 and 2021 were selected. A total of 35 articles, 2 dissertations, 2 theses and 1 monograph were found with a research focus on the topic of interest. The existence of other hosts, besides man, was described, mainly armadillos of the species Dazypus novemcinctus, helping to analyze the situation of territoriality, and to delimit the characteristics of the fungus ecosystem. Social and environmental changes also influence the incidence of the disease. There is a strong association in the epidemiological pattern and ecosystem of Paracoccidioides, through this analysis it is possible to predict possible outbreaks and control the disease.
\end{abstract}

Keywords: Ecoepidemiology; Epidemiology; Ecology; Paracoccidioidomycosis.

\section{Resumen}

La paracoccidioidomicosis es una micosis sistémica prevalente en América Latina, es causada por Paracoccidioides brasiliensis y Paracoccidioides lutzii, los cuales son hongos termodimórficos, la fase filamentosa está presente en el suelo de manera saprofita. El objetivo es presentar datos ecoepidemiológicos que relacionen factores ecológicos y socioambientales con la enfermedad. Se realizó una revisión narrativa a través de las bases de datos PubMed, Scielo, LILACS, MEDLINE y Google Scholar, donde se seleccionaron artículos, tesis y disertaciones que abordaron el tema entre 2000 y 2021. Se encontraron 35 artículos, 2 disertaciones, 2 tesis y 1 monografía con un enfoque de 
investigación en el tema de interés. Se describió la existencia de otros hospedadores, además del hombre, principalmente armadillos de la especie Dazypus novemcinctus, ayudando a analizar la situación de territorialidad y delimitar las características del ecosistema fúngico. Los cambios sociales y ambientales también influyen en la incidencia de la enfermedad. Existe una fuerte asociación en el patrón epidemiológico y ecosistema de Paracoccidioides, a través de este análisis es posible predecir posibles brotes y controlar la enfermedad.

Palabras clave: Ecoepidemiología; Epidemiología; Ecología; Paracoccidioidomicosis.

\section{Introdução}

Infecções fúngicas invasivas são uma das maiores causas de mortes no mundo, são causadas principalmente por micoses de infecção sistêmica, resultado da inalação de esporos de fungos dimórficos que frequentemente iniciam as infecções no pulmão e podem se disseminar para outros tecidos. A paracoccidiodomicose (PCM) é uma micose sistêmica que representa um importante problema para saúde pública, segundo o Ministério da Saúde ela representa uma das dez principais causas de morte por doenças infecciosas e parasitarias, crônica e recorrente no Brasil (Moreira, 2008).

É uma infecção crônica e granulomatosa, causada por duas principais espécies, o Paracoccidioides brasiliensis e o Paracoccidioides lutzii, fungos termodimórficos. A fase filamentosa destes fungos habita solos férteis e ricos em vegetação, de forma saprofítica e o homem se infecta ao inalar conídios ou hifas liberadas pelo fungo, afetando principalmente trabalhadores rurais do sexo masculino de meia idade. Dependendo da quantidade de conídios inalados, tempo de exposição e condições imunológicas, a doença poderá ou não se instalar (Faria et al., 2004).

A PCM é uma micose endêmica e prevalente na América Latina, há casos da Argentina ao México, os países com maiores números de casos são Brasil, Venezuela e Colômbia, respectivamente (Angela Restrepo, Benard, de Castro, Agudelo, \& Tobón, 2008). No Brasil, micoses sistêmicas não fazem parte da lista nacional de agravos de notificação compulsória, só alguns Estados do país fazem a vigilância epidemiológica da infecção, ou seja, não há dados precisos sobre a doença, e a coleta de dados para a compreensão epidemiológica é baseado em estudos de caso, dados e informações hospitalares e do Sistema Único de Saúde e estudos de isolamentos do microrganismo e suas linhagens (Millington et al., 2018). Baseado em análise clínica e epidemiológica de estudo de Hahn et al., 2019 no Estado do Mato Grosso, Brasil não é possível, até agora estabelecer as diferenças clínicas importantes que podem ser atribuídos a P. lutzii ou P. brasiliensis (Hahn et al., 2019).

O objetivo deste trabalho é apresentar os dados ecoepidemiológicos associando fatores ecológicos e socioambientais à doença.

\section{Metodologia}

De natureza qualitativa (Flick, 2009; Patton, 2001) esta pesquisa caracteriza-se como revisão bibliográfica do tipo narrativa (Gil, 2008). Realiza uma análise dos aspectos ecoepidemiológicos de Paracoccidioides spp. Foram selecionados artigos, teses, dissertações e monografias dos bancos de dados PubMed, Scielo, LILACS, MEDLINE e Google Scholar. Os termos utilizados para a busca foram em conjunto com o Operador Booleano "AND": "Ecoepidemiologia"; "Ecologia"; "Epidemiologia"; "Paracoccidioidomicose".

Como critério de inclusão foram selecionados materiais dos tipos de revisões sistemáticas, metanálises, estudos de coorte, estudos de caso, séries de caso, caso controle, estudo randomizado, experimentais, revisões narrativas e integrativas, estudos longitudinais, transversais e ecológicos que abordassem a temática da biologia e ecologia do fungo, em idiomas português, inglês e espanhol, com o texto disponível de forma completa e na integra, disponíveis gratuitamente. Foi utilizado o corte temporal entre 2000 e 2021. Foram excluídos artigos que abordassem a patologia sem pertinência com o assunto pesquisado. Chegou-se no final dos critérios de levantamento de dados ao número de 35 artigos, 2 teses, 2 dissertações e 1 monografia. 


\section{Resultados}

\subsection{Fatores ecológicos}

Muitos estudos no Brasil e Colômbia têm trazido dados positivos sobre isolamento de $P$. brasiliensis das vísceras do tatu silvestre, de uma espécie em especial (Dazypus novemcinctus), os quais através de metodologias semelhantes sob licença ambiental demonstram de 20 a $75 \%$ de presença do fungo. A partir de exames com os animais investigados pode-se ter achados de que estes tinham a doença em si e não apenas a infecção. Na natureza, a distribuição geográfica de D. novemcinctus se sobrepõe muito às áreas onde a doença é prevalente, e é um animal que está em contato próximo com o solo. Seus hábitos de vida mantêm o solo e esse em contato entre si e, desse modo, podem ser divididos com mais precisão a área de possível armazenamento de $P$. brasiliensis contribuindo para determinar a região que o paciente se contamina (Naiff et al., 1986 apud Fernandes, Deps, Tomimori-Yamashita, \& Camargo, 2004; Marques, 2003).

Costa et al., 1995 realizaram um inquérito epidemiológico no qual 96 animais silvestres capturados no campo, sob licença ambiental foram submetidos à investigação de Paracoccidioidomicose e Histoplasmose (Costa et al., 1995 apud Martinez, 2015). Os resultados apontam que houve cerca de 52\% de positividade para paracoccidioidina e, havendo, em $80 \%$ deles, concomitante positividade para histoplasmina. As observações realizadas foram que houve maior taxa de positividade para paracoccidioidina em animais de hábitos terrestres, nas famílias Felidae e Procyonidae da ordem Carnivora em comparação a aqueles de hábitos de vida arbórea da ordem Primates. A esses achados somam-se os anteriormente relatados de isolamento do P. brasiliensis em excrementos de morcego, Artibeus lituratus, na Colômbia em 1965, em vísceras de símio, Saimiri sciureus, na Bolívia em 1977 e em fezes de pinguim, Pygoscelis adeliae, na Antártida Uruguaiana em 1989 (Grose \& Tamsitt, 1965 apud Bocca et al., 2013; Garcia et al., 1993 apud Marques, 2003; Johnson \& Lang, 1977 apud Teixeira et al., 2014). Em 2002 foi registrado o primeiro caso de paracoccidioidomicose canina(Ricci et al., 2004). Os cães que vivem em área rural apresentam uma taxa de infecção mais alta por PCM que os que vivem em área urbana (Fontana et al., 2010). Coelhos criados em ambientes livres têm taxas de infecção mais elevadas do que animais enjaulados e cerca de $80 \%$ dos coelhos expostos à infecção natural desenvolvem anticorpos contra P. brasiliensis (Belitardo et al., 2014). Dessa maneira, há maior conhecimento sobre a existência de outros hospedeiros, além do homem, assim como a situação da territorialidade em que o $P$. brasiliensis encontra-se em forma de saprófito. Deve-se levar em consideração que pesquisas demonstram a dificuldade de isolamento do fungo do solo para estudos epidemiológicos (Ono, Itano, Mizuno, Mizuno, \& Camargo, 2002).

Estudos mais recentes, com a utilização de técnicas moleculares foi avaliado a presença de infecção por $P$. brasiliensis em 19 animais selvagens mortos em estradas de áreas endêmicas, das espécies Cavia aperea, Cerdocyon thirty, Dasypus novemcinctus, Dasypus $\quad$ septemcinctus, Didelphis albiventris, Eira barbara, Gallictis vittata, Procyon cancrivorus e Sphiggurus spinosus. Foi encontrado amplicons específicos para o fungo em variados órgãos das diferentes espécies, corroborando com a ideia de diversificados perfis de disseminação, indicando diferentes interações com o $P$. brasiliensis (Richini-Pereira et al., 2008).

Paracoccidioidomicose disseminada também foi detectada em uma preguiça-de-dois-dedos que morreu após ser capturada na Guiana, fato que demonstra a endemicidade desta região (Trejo-Chávez, Ramírez-Romero, Ancer-Rodríguez, Nevárez-Garza, \& Rodríguez-Tovar, 2011). Estruturas semelhantes às de $P$. brasiliensis foram observadas no fígado de um macaco-esquilo capturado na Bolívia (Johnson \& Lang, 1977 apud Teixeira et al., 2014). Levantamentos sorológicos ou testes cutâneos com antígenos de $P$. brasiliensis revelaram a existência de paracoccidioidomicose-infecção em gatos, cães, galinhas, porcos, bovinos, equinos, ovinos, caprinos, coelhos, em macacos e em outros animais silvestres livres ou cativos. Porém, em testes sorológicos ou cutâneos, o exame anatomopatológico de animais reativos a antígenos fúngicos não revelou danos 
causados por P. brasiliensis (Costa et al., 1995 apud Martinez, 2015). Isso indica que, com exceção dos tatus, a infecção apresenta baixo risco de espalhamento de fungos e doenças dos animais.

Nas áreas onde o fungo vive na natureza, o ser humano (paciente) contrai a infecção na fase de levedura (Angela Restrepo, Gómez, \& Tobón, 2012). Essas áreas são áreas de floresta tropical ou subtropical com abundantes cursos de água, temperaturas entre $10^{\circ}$ a $28^{\circ} \mathrm{C}$, pluviosidade elevada entre 500 e $2500 \mathrm{~mm}$ e solo ácido e fértil (A. Restrepo, McEwen, \& Castañeda, 2001). Essas condições são semelhantes às da Venezuela, São Paulo e Minas Gerais, onde o fungo foi isolado (Fontana et al., 2010). Ademais podem ser encontradas em rios da selva amazônica e pequenas florestas indígenas no Uruguai. Paracoccidioides brasiliensis foi encontrado no solo dessas áreas (Hrycyk, 2018).

Um estudo anterior mostrou que a umidade do solo, associada a disponibilidade de água e presença de vegetação favorece a esporulação e dispersão de Paracoccidioides spp. (Shikanai-Yasuda et al., 2017). Apesar da infecção, o tipo de solo parece não ter muito efeito. Em humanos, porém, a infecção ocorre mais geralmente por solos argilosos (Bagagli, Theodoro, Bosco, \& McEwen, 2008).

Foi constatado por Bagagli et al., 2008 que as condições climáticas associadas a textura do solo determinam o crescimento do patógeno em sua fase saprofítica (Bagagli et al., 2008). Foi observado também por Arantes et al., 2016 que a presença de alumínio no solo interfere na sobrevivência do fungo, em grandes quantidades pode limitar ou inibir o crescimento do fungo, estudos feitos em tatus que habitam regiões de solo rico em alumínio não apresentavam contaminação pelo Paracoccidioides (Arantes, Theodoro, Teixeira, Bosco, \& Bagagli, 2016). O fungo está refém dos fatores de seleção ambientais, como radiação solar, precipitação, competição, predação, textura e compostos químicos no solo (Figura 1) (Gomes, 2014).

Figura 1 - Ciclo de vida hipotético do Paracoccidioides brasiliensis.

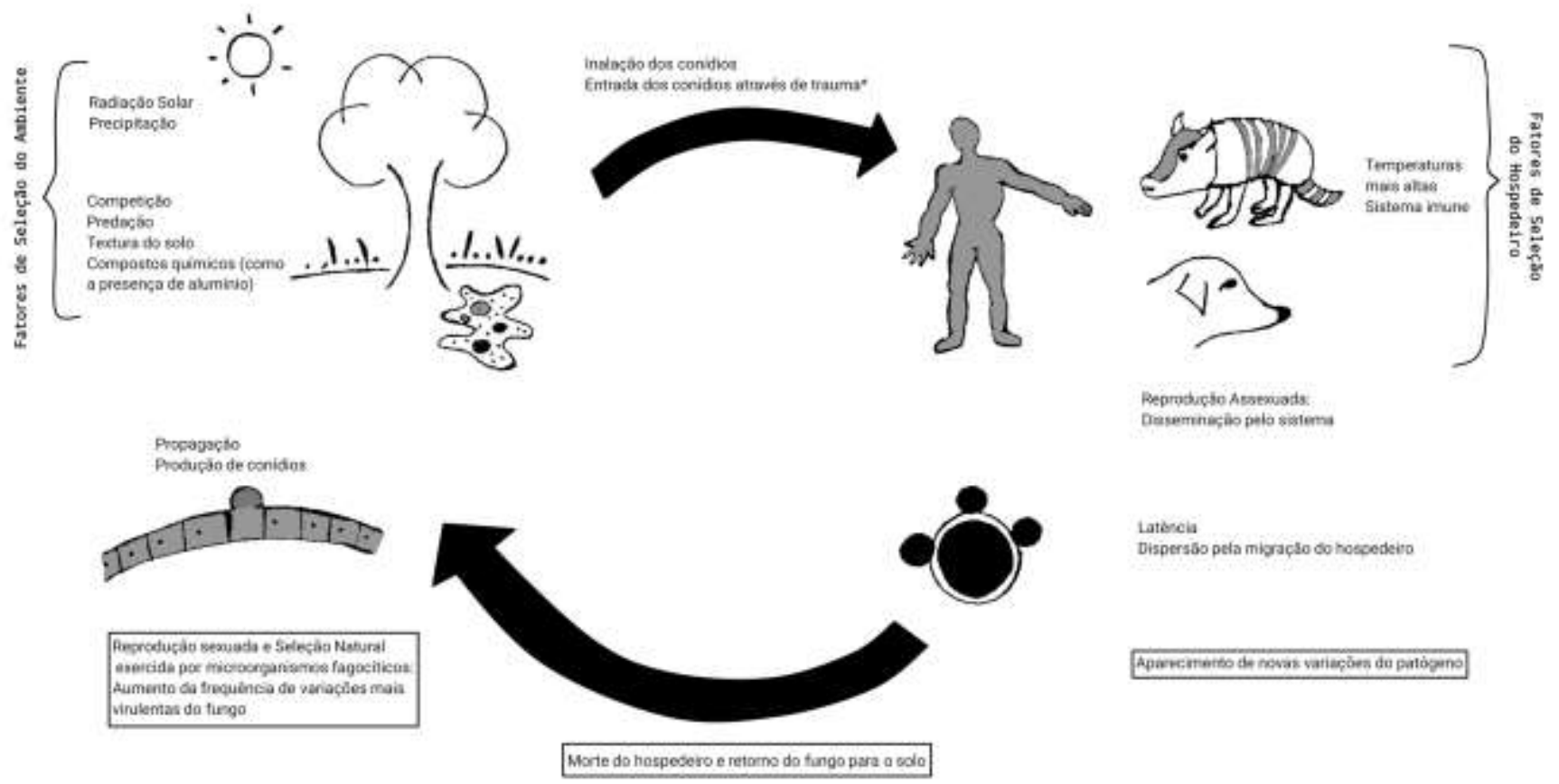




\subsection{Fatores socioambientais}

É comentada a alta prevalência da micose em crianças em áreas endêmicas da Região Amazônica. O isolamento de Paracoccidioides brasiliensis de vísceras de tatus (Dasypus novemcinctus) no Pará demonstra que o fungo tem raiz indígena na Região Amazônica (Fonseca et al., 1999 apud Shikanai-Yasuda, Telles Filho, Mendes, Colombo, \& Moretti, 2006). Em 1971, Campbell predisse que à medida que o homem avançasse, disturbando o solo, em regiões onde o fungo é nativo, a PCM constituiria uma ameaça cada vez maior (Fonseca et al., 1999 apud Shikanai-Yasuda et al., 2006). Tal está acontecendo em vastas áreas dos Estados do Pará, Maranhão e Tocantins, colonizados nas três últimas décadas. Na região Norte é provável, também, que outras formas clínicas da micose possam ser observadas em crianças (Shikanai-Yasuda et al., 2017). O desmatamento acelerado, revolvimento do solo para o plantio e pecuária determinam a suspensão do fungo na atmosfera, contribuindo para formação de áreas hiperendêmicas, com a maior taxa de infecção de adultos jovens (Almeida, 2014).

Há indícios de que mudanças socioambientais estejam associadas à epidemiologia da doença. Foi observada redução progressiva do número de casos da forma aguda/subaguda da paracoccidioidomicose no Estado de Mato Grosso do Sul, o que pode ter sido consequência da política de controle público e redução do trabalho infantil (Fabris et al., 2014). Desse modo, elenca-se abaixo os eventos ocorridos nos últimos anos que tiveram relação determinante com a alta na incidência da PCM (Tabela 1).

Tabela 1 - Relação das mudanças socioambientais com elevação da incidência da doença organizada por cada evento ocorrido, período, região e consequências.

\begin{tabular}{|c|c|c|c|c|}
\hline Evento & $\begin{array}{l}\text { Cidade/ Estado/ } \\
\text { Região/ País }\end{array}$ & $\begin{array}{l}\text { Período do } \\
\text { evento do } \\
\text { estudo } \\
\end{array}$ & Consequências & Referências \\
\hline 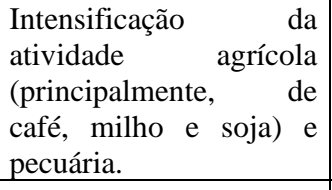 & Rondônia, Brasil. & $2015-2017$. & $\begin{array}{l}\text { Registrou-se predomínio da doença } \text { na região } \\
\text { central do Estado com } 176 \text { casos de } \\
\text { paracoccidioidomicose em uma incidência de } 9-40 \\
\text { casos / } 100000 \text { habitantes / ano. }\end{array}$ & (Pestana, 2019) \\
\hline $\begin{array}{l}\text { El Niño Southern } \\
\text { Oscilation (ENSO). }\end{array}$ & $\begin{array}{l}\text { São Paulo, Brasil } \\
\text { (compreendendo } \\
44 \quad \text { municípios } \\
\text { das r região } \\
\text { centro-oeste do } \\
\text { Estado). }\end{array}$ & $1982-1983$. & $\begin{array}{l}\text { Foram registrados no período do estudo (de } 1966 \text { a } \\
\text { 2006) } 825 \text { casos com diagnóstico confirmado de } \\
\text { PCM. O teste temporal retratou um cluster } \\
\text { significativo em } 1985 \text { (P <0,005). Este cluster } \\
\text { compreendeu } 10 \text { casos, embora fossem esperados } \\
2,19 \text { para este ano nesta área. A infecção foi } \\
\text { estimada para ter ocorrido } 1-2 \text { anos antes e através } \\
\text { de múltiplas análises de regressão determinou que a } \\
\text { variabilidade do clima foi um fator significativo. }\end{array}$ & $\begin{array}{l}\text { (Barrozo et al., 2010; } \\
\text { do Valle et al., 2017) }\end{array}$ \\
\hline 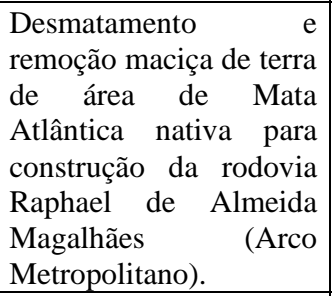 & $\begin{array}{l}\text { Microrregião da } \\
\text { Baixada } \\
\text { Fluminense, Rio } \\
\text { de Janeiro, } \\
\text { Brasil. }\end{array}$ & $2015-2016$. & $\begin{array}{l}\text { A incidência de paracoccidioidomicose aguda na } \\
\text { área afetada após a construção da rodovia }(8,25 \\
\text { casos / } 1 \text { milhão de pessoas / ano) foi maior do que } \\
\text { antes da construção da rodovia (1,29 casos / } 1 \\
\text { milhão de pessoas / ano). }\end{array}$ & $\begin{array}{l}\text { (do Valle et al., } \\
\text { 2017) }\end{array}$ \\
\hline $\begin{array}{ll}\text { Desmatamento } & \text { na } \\
\text { microrregião } & \text { da } \\
\text { barragem de Itaipu. } & \end{array}$ & $\begin{array}{l}\text { Região do lago } \\
\text { de Itaipu, Paraná, } \\
\text { Brasil. }\end{array}$ & $\begin{array}{l}2008-2009 \\
(18 \text { meses })\end{array}$ & $\begin{array}{l}\text { Registrou-se } 102 \text { casos da doença e } 15 \text { óbitos dos } \\
\text { pacientes, determinando, assim, a região como } \\
\text { endêmica e de elevada taxa de mortalidade. }\end{array}$ & $\begin{array}{l}\text { (do Valle et al., } \\
\text { 2017; Loth, Castro, } \\
\text { Silva, \& Gandra, } \\
\text { 2011) }\end{array}$ \\
\hline $\begin{array}{l}\text { El Niño Southern } \\
\text { Oscilation (ENSO). }\end{array}$ & $\begin{array}{l}\text { Nordeste } \quad \text { da } \\
\text { Argentina. }\end{array}$ & $2010-2012$ & $\begin{array}{l}\text { Relatou-se } 6 \text { casos agudos/ subagudos de PCM em } \\
\text { crianças (de média e mediana de idade de } 12 \text { anos). }\end{array}$ & $\begin{array}{l}\text { (Giusiano et al., } \\
\text { 2019) }\end{array}$ \\
\hline
\end{tabular}




\section{Discussão}

O perfil epidemiológico da doença em áreas endêmicas está relacionado e delimitado pelas características de seu ecossistema. Inúmeros animais apresentaram em estudos antígenos contra $P$. brasiliensis, porém, apenas os tatus parecem desenvolver a infecção. Os tatus mostram evidências de grande valor sobre a cadeia epidemiológica dela, pois estes convivem em condições geográficas semelhantes às do fungo e estão em contato direto com o solo em virtude de seus hábitos de sobrevivência e por este ser um animal sem hábitos migratórios. Os tatus infectados costumam ficar em áreas sombreadas próximas aos rios, e há solos argilosos e arenosos, o que indica que a umidade é um fator importante no crescimento dos fungos (Simões, Marques, \& Bagagli, 2004). Em outro estudo de Brito, 2012 observou-se de novo o desenvolvimento do Paracoccidioides em solos argilosos e arenosos, desde que a umidade desses solos seja elevada (Brito, 2012).

Há registros de infecções por Paracoccidioides spp. depois de distúrbios do solo que resultaram na disseminação aérea de propágulos fúngicos (Angela Restrepo et al., 2012). Levando em conta a ecologia do fungo, as suas cepas têm alta relação com plantações de café e tabaco que aparentemente envolvem um risco maior de inalação de conídios de $P$. brasiliensis presentes no solo (Silva-Vergara \& Martínez, 1998 apud Bellissimo-Rodrigues, Machado, \& Martinez, 2011; Calle et al., 2001; Torrado, Castañeda, Hoz, \& Restrepo, 2000). Há indícios que a mecanização agrícola, uso de produtos químicos nas plantações (como herbicidas) e atividade pecuarista tornem o indivíduo passível de contaminação pelo fungo, já que envolvem o manejo e contato com solo (Moreira, 2008).

A relevância do estudo sobre a ecologia traz à tona o empasse da real incidência de cada espécie filogenética e seu envolvimento na prática clínica e epidemiológica. Desse modo, devem ser feitos outros estudos em diferentes regiões do Brasil e da América Latina para comparar as espécies de PCM, as formas e manifestações clínicas com o perfil genético dessas entidades. Além de que as manifestações das formas clínicas incomuns em áreas endêmicas, a formação de áreas hiperendêmicas, de novas áreas endêmicas e/ou incidência de novos casos resultantes de alterações socioambientais deve ser considerada como importante fator. De maneira que pode ser levantada discussão da associação socioambiental com a doença nos tópicos no Brasil e na América Latina, abordados abaixo.

\subsection{No Brasil}

Fatores ambientais, resultantes da expansão agropecuária e da derrubada de florestas, sobretudo na Amazônia, também contribuíram para o atual panorama da micose (Corte et al., 2012; Forjaz et al., 1999 apud do Valle et al., 2017; "Guia de Vigilância Epidemiológica CAD. 7 Ed. Brasília/DF: Ministério Da Saúde; 2009.,” 2009; Vieira, Alves, Lima, Camargo, \& Sousa, 2014). Forjaz et al. (1999) em seu estudo sobre PCM em uma população indígena observaram que a doença também estava presente na população indígena Suruí, em Rondônia, os quais mudaram seus hábitos de subsistência e passaram a trabalhar com a cultura do café (Forjaz et al., 1999 apud do Valle et al., 2017). Testes cutâneos demonstraram que a infecção por $P$. brasiliensis foi mais comum nesta tribo do que em tribos vizinhas não envolvidas com atividades agrícolas (Toyotani \& Souza, 2019).

A razão do predomínio da prevalência da doença na região central de Rondônia é a aceleração do desenvolvimento agropecuário, que coincide com a área de plantios agrícolas como milho, café, soja e pecuária (Toyotani \& Souza, 2019). Esse pode ser um dos motivos para isso, pois a literatura relaciona casos de PCM a trabalhos em áreas rurais, enfatizando as características climáticas existentes em Rondônia, água pluvial abundante e florestas úmidas que são benéficas (Pestana, 2019).

Sabe-se que variáveis ambientais e / ou climáticas afetam a incidência de doenças fúngicas endêmicas (Klein et al., 1987 apud Chapman et al., 2008; Comrie, 2005; Kolivras \& Comrie, 2003). No Brasil, o comportamento do ENSO explica 
grande parte da variabilidade interanual das chuvas próximo ao equador e no sul (Kayano \& Andreoli, 2007). Essa retenção incomum de água no solo em 1982/1983 foi causada pelo evento El Niño mais forte na área nos últimos 50 anos, que também levou a chuvas anormais, o qual fez com que a precipitação na área de São Paulo fosse mais de dois desvios padrão acima da média (Fergusson, Orsi, \& Heinl, 2013).O fenômeno El Niño está relacionado à disseminação de várias doenças infecciosas (Colombo, Tobón, Restrepo, Queiroz-Telles, \& Nucci, 2011). No entanto, na PCM, além da dificuldade em identificar clusters desta doença, a ligação com outros eventos El Niño nem sempre é óbvia, pois não existem dois eventos ENSO exatamente iguais, em termos de intensidade, organização temporal e espacial, e clima temperado. Os eventos são diferentes. A resposta ecológica varia de evento para evento (Fergusson et al., 2013).

Relata-se o primeiro surto de paracoccidioidomicose após desmatamento e remoção maciça de terra durante um projeto de urbanização (construção de uma rodovia, a Rodovia do Arco Metropolitano) no Rio de Janeiro, Brasil. Em 2016, após a construção de um anel rodoviário na Baixada Fluminense, área metropolitana do Rio de Janeiro houve extensa derrubada de vegetação acompanhada de grande movimentação de terra (do Valle et al., 2017). Fato semelhante pode ter ocorrido no Sul do Brasil na microrregião da barragem de Itaipu, onde foram registrados 102 casos de paracoccidioidomicose em um período de 18 meses (Loth et al., 2011).

\subsection{Na América Latina}

As populações indígenas nativas da América Latina mudaram suas antigas práticas de subsistência para cultivar café após o desmatamento da floresta amazônica com a abertura de novas fronteiras agrícolas, que resultou em infecções por paracoccidioidomicose (Coimbra Júnior et al., 1994 apud Colombo et al., 2011; A. C. do Valle et al., 1991 apud do Valle et al., 2017).

Em 2009, o fenômeno ENSO afetou a área do Nordeste da Argentina (NEA), com maior precipitação ("Perspectivas de Cosechas y Situación Alimentaria, No.4,Noviembre 2009,” 2009). Além desse importante impacto climático registrado, mudanças causadas pelo homem junto com mudanças ecológicas também foram introduzidas na NEA nas últimas décadas. Devido ao desmatamento, às mudanças nas práticas agrícolas e à construção e operação de uma das maiores hidrelétricas da América do Sul, Yacyretá, a área enfrenta mudanças ecológicas e também se tornou endêmica para a micose (Null, 2017).

\section{Conclusão}

A mudança no padrão epidemiológico da paracoccidioidomicose está delimitada pelo ecossistema e, desse modo são necessárias pesquisas geoclimáticas para prever novos possíveis surtos dela e uma proposta de intervenção do trabalho é o controle da doença por meio de geoprocessamento considerando a relativa distribuição do tatu silvestre e sua relação com a dispersão fúngica geográfica. Além disso, a ocorrência de diferentes espécies e classificações filogenéticas de Paracoccidioides também pode favorecer para a mudança do perfil epidemiológico.

Recomenda-se novos estudos epidemiológicos observacionais ou experimentais, exploratórios, dos tipos comparativos, ecológicos, estudos caso-controle, de coorte, de caráter qualitativo ou quantitativo seja prospectivo ou retrospectivo para que possa ser estabelecido o grau de relação ecoepidemiológico entre os fatores citados e possíveis novas associações socioambientais à doença para prospecção de novas medidas preventivas.

\section{Referências}

Almeida, H. L. de. (2014). Paracoccidioidomicose: Perfil epidemiológico, clínico e terapêutico dos pacientes diagnosticados nos serviços de referência do Estado de Mato Grosso (2006-2013) (Thesis, Universidade Federal de Mato Grosso). Universidade Federal de Mato Grosso. http://ri.ufmt.br/handle/1/489 
Arantes, T. D., Theodoro, R. C., Teixeira, M. de M., Bosco, S. de M. G., \& Bagagli, E. (2016). Environmental Mapping of Paracoccidioides spp. In Brazil Reveals New Clues into Genetic Diversity, Biogeography and Wild Host Association. PLoS Neglected Tropical Diseases, $10(4)$, e0004606. 10.1371/journal.pntd.0004606

Bagagli, E., Theodoro, R. C., Bosco, S. M. G., \& McEwen, J. G. (2008). Paracoccidioides brasiliensis: Phylogenetic and ecological aspects. Mycopathologia, 165(4-5), 197-207. 10.1007/s11046-007-9050-7

Barrozo, L. V., Benard, G., Silva, M. E. S., Bagagli, E., Marques, S. A., \& Mendes, R. P. (2010). First Description of a Cluster of Acute/Subacute Paracoccidioidomycosis Cases and Its Association with a Climatic Anomaly. PLOS Neglected Tropical Diseases, 4(3), e643. 10.1371/journal.pntd.0000643

Belitardo, D. R., Calefi, A. S., Sbeghen, M. R., de Oliveira, G. G., Watanabe, M. A. E., de Camargo, Z. P., \& Ono, M. A. (2014). Paracoccidioides brasiliensis infection in domestic rabbits (Oryctolagus cuniculus). Mycoses, 57(4), 222-227. 10.1111/myc.12146

Bellissimo-Rodrigues, F., Machado, A. A., \& Martinez, R. (2011). Paracoccidioidomycosis epidemiological features of a 1,000-cases series from a hyperendemic area on the southeast of Brazil. The American Journal of Tropical Medicine and Hygiene, 85(3), 546-550. PubMed (21896820). 10.4269/ajtmh.2011.11-0084

Bocca, A. L., Amaral, A. C., Teixeira, M. M., Sato, P. K., Shikanai-Yasuda, M. A., \& Soares Felipe, M. S. (2013). Paracoccidioidomycosis: Ecoepidemiology, taxonomy and clinical and therapeutic issues. Future Microbiology, 8(9), 1177-1191. 10.2217/fmb.13.68

Brito, M. M. dos S. (2012). Morfologia, virulência e perfil genético de Paracoccidioides brasiliensis: Avaliação pré e pós-passagem em modelo murino de isolados provenientes de forma clínica crônica humana da paracoccidioidomicose (Thesis). https://www.arca.fiocruz.br/handle/icict/8049

Calle, D., Rosero, D. S., Orozco, L. C., Camargo, D., Castañeda, E., \& Restrepo, A. (2001). Paracoccidioidomycosis in Colombia: An ecological study. Epidemiology and Infection, 126(2), 309-315. 10.1017/s0950268801005052

Chapman, S. W., Dismukes, W. E., Proia, L. A., Bradsher, R. W., Pappas, P. G., Threlkeld, M. G., \& Kauffman, C. A. (2008). Clinical Practice Guidelines for the Management of Blastomycosis: 2008 Update by the Infectious Diseases Society of America. Clinical Infectious Diseases, 46(12), 1801-1812. $10.1086 / 588300$

Colombo, A. L., Tobón, A., Restrepo, A., Queiroz-Telles, F., \& Nucci, M. (2011). Epidemiology of endemic systemic fungal infections in Latin America. Medical Mycology, 49(8), 785-798. 10.3109/13693786.2011.577821

Comrie, A. C. (2005). Climate factors influencing coccidioidomycosis seasonality and outbreaks. Environmental Health Perspectives, 113(6), 688-692. PubMed (15929890). 10.1289/ehp.7786

Corte, A. C., Gennari, S. M., Labruna, M. B., Camargo, L. M. A., Itano, E. N., Freire, R. L., \& Ono, M. A. (2012). Paracoccidioides brasiliensis infection in dogs from Western Brazilian Amazon. Pesquisa Veterinária Brasileira, 32, 649-652. 10.1590/S0100-736X2012000700011

do Valle, A. C. F., Marques de Macedo, P., Almeida-Paes, R., Romão, A. R., Lazéra, M. D. S., \& Wanke, B. (2017). Paracoccidioidomycosis after Highway Construction, Rio de Janeiro, Brazil. Emerging Infectious Diseases, 23(11), 1917-1919. 10.3201/eid2311.170934

Fabris, L. R., Andrade, Ú. V., Santos, A. F. D., Marques, A. P. D. C., Oliveira, S. M. D. V. L. D., Mendes, R. P., \& Paniago, A. M. M. (2014). Decreasing Prevalence Of The Acute/Subacute Clinical Form Of Paracoccidioidomycosis In Mato Grosso Do Sul State, Brazil. Revista Do Instituto de Medicina Tropical de São Paulo, 121. 10.1590/S0036-46652014000200006

Faria, E. C., Pessoa-Júnior, V. P., De-Maria-Moreira, N. L., Silva De Amorim, D., Gomes, A. P., Capone, D., \& Siqueira-Batista, R. (2004). Micoses pulmonares. Revista Brasileira de Medicina, 61(8), 511-526. 10.12957/rhupe.2010.9179

Fergusson, E., Orsi, J. A., \& Heinl, S. C. (2013). Proceedings of the 25th Northeast Pacific Pink and Chum Salmon Workshop 2012, Centennial Hall, Juneau, AK, 13-15 February 2012. Retrieved from https://repository.library.noaa.gov/view/noaa/4572

Fernandes, G. F., Deps, P., Tomimori-Yamashita, J., \& Camargo, Z. P. (2004). IgM and IgG antibody response to Paracoccidioides brasiliensis in naturally infected wild armadillos (Dasypus novemcinctus). Medical Mycology, 42(4), 363-368. 10.1080/13693780310001658748

Flick, U. (2009). Qualidade na pesquisa qualitativa. Artmed.

Fontana, F. F., dos Santos, C. T. B., Esteves, F. M., Rocha, A., Fernandes, G. F., do Amaral, C. C., ... Silva-Vergara, M. L. (2010). Seroepidemiological survey of paracoccidioidomycosis infection among urban and rural dogs from Uberaba, Minas Gerais, Brazil. Mycopathologia, 169(3), 159-165. $10.1007 / \mathrm{s} 11046-009-9241-5$

Gil, A. C. (2008). Métodos e técnicas de pesquisa social. Atlas.

Giusiano, G., Aguirre, C., Vratnica, C., Rojas, F., Corallo, T., Cattana, M. E., \& de Los Angeles Sosa, M. (2019). Emergence of acute/subacute infant-juvenile paracoccidioidomycosis in Northeast Argentina: Effect of climatic and anthropogenic changes? Medical Mycology, 57(1), 30-37. 10.1093/mmy/myx153

Gomes, D. M. de A. (2014). Estudo sobre a ecologia e origem da virulência de Paracoccidioides brasiliensis—Caracterização de sua interação com Acanthamoeba castellanii (Thesis). Retrieved from https://repositorio.unb.br/handle/10482/16085

Guia de Vigilância Epidemiológica CAD. 7 ed. Brasília/DF: Ministério da Saúde; 2009. (2009). Retrieved July 4, 2021, from https://bvsms.saude.gov.br/bvs/publicacoes/guia_vigilancia_epidemiologica_7ed.pdf 
Hahn, R. C., Rodrigues, A. M., Della Terra, P. P., Nery, A. F., Hoffmann-Santos, H. D., Góis, H. M., \& de Camargo, Z. P. (2019). Clinical and epidemiological features of paracoccidioidomycosis due to Paracoccidioides lutzii. PLoS Neglected Tropical Diseases, 13(6), e0007437-e0007437. PubMed (31163028). 10.1371/journal.pntd.0007437

Hrycyk, M. F. (2018). Ecologia de Paracoccidioides brasiliensis e Paracoccidioides lutzii e sua associação com o tatu Dasypus novemcinctus nos estados de São Paulo e Mato Grosso, Brasil. (Thesis). https://repositorio.unesp.br/bitstream/handle/11449/153222/hrycyk_mf_dr_bot.pdf?sequence=3\&isAllowed=y

Kayano, M. T., \& Andreoli, R. V. (2007). Relations of South American summer rainfall interannual variations with the Pacific Decadal Oscillation. International Journal of Climatology, 27(4), 531-540. 10.1002/joc. 1417

Kolivras, K. N., \& Comrie, A. C. (2003). Modeling valley fever (coccidioidomycosis) incidence on the basis of climate conditions. International Journal of Biometeorology, 47(2), 87-101. 10.1007/s00484-002-0155-x

Loth, E. A., Castro, S. V. de, Silva, J. R. da, \& Gandra, R. F. (2011). Occurrence of 102 cases of paracoccidioidomycosis in 18 months in the Itaipu Lake region, Western Paraná. Revista Da Sociedade Brasileira de Medicina Tropical, 44, 636-637. 10.1590/S0037-86822011000500023

Marques, S. A. (2003). Paracoccidioidomicose: Atualização epidemiológica, clínica e terapêutica. Anais Brasileiros de Dermatologia, 78(2), 135-146. $10.1590 /$ S0365-05962003000200002

Martinez, R. (2015). Epidemiology Of Paracoccidioidomycosis. Revista Do Instituto de Medicina Tropical de São Paulo, 57, 11-20. 10.1590/S003646652015000700004

Millington, M. A., Nishioka, S. de A., Martins, S. T., Santos, Z. M. G. dos, Lima, F. E. F. de, \& Alves, R. V. (2018). Paracoccidioidomicose: Abordagem histórica e perspectivas de implantação da vigilância e controle. Epidemiologia e Serviços de Saúde, 27. 10.5123/S1679-49742018000500002

Moreira, A. P. V. (2008). Paracoccidioidomicose: Histórico, etiologia, epidemiologia, patogênese, formas clínicas, diagnóstico laboratorial e antígenos. BEPA, Bol. epidemiol. paul. (Online), 5, 14.

Null, J. (2017). El Nino and La Nina years and intensities based on Oceanic Nino index (ONI). Retrieved July 7, 2021, from https://ggweather. com/enso/oni. htm

Ono, M. A., Itano, E. N., Mizuno, L. T., Mizuno, E. H. F., \& Camargo, Z. P. (2002). Inhibition of Paracoccidioides brasiliensis by pesticides: Is this a partial explanation for the difficulty in isolating this fungus from the soil? Medical Mycology, 40(5), 493-499. 10.1080/mmy.40.5.493.499

Patton, M. Q. (2001). Qualitative research and evaluation methods. Sage Publications.

Perspectivas de Cosechas y Situación Alimentaria, No.4,noviembre 2009. (2009). http://www.fao.org/3/ak340s/ak340s05b.htm

Pestana, L. G. R. (2019). Incidência de paracoccidioidomicose no estado de Rondônia de 2015 a 2017 (Thesis). http://repositorio.saolucas.edu.br:8080/xmlui/handle/123456789/2946

Restrepo, A., McEwen, J. G., \& Castañeda, E. (2001). The habitat of Paracoccidioides brasiliensis: How far from solving the riddle? Medical Mycology, 39(3), 233-241. 10.1080/mmy.39.3.233.241

Restrepo, Angela, Benard, G., de Castro, C. C., Agudelo, C. A., \& Tobón, A. M. (2008). Pulmonary paracoccidioidomycosis. Seminars in Respiratory and Critical Care Medicine, 29(2), 182-197. 10.1055/s-2008-1063857

Restrepo, Angela, Gómez, B. L., \& Tobón, A. (2012). Paracoccidioidomycosis: Latin America's own fungal disorder. Current Fungal Infection Reports, 6(4), 303-311. 10.1007/s12281-012-0114-x

Ricci, G., Mota, F. T., Wakamatsu, A., Serafim, R. C., Borra, R. C., \& Franco, M. (2004). Canine paracoccidioidomycosis. Medical Mycology, 42(4), 379383. $10.1080 / 1369378032000141417$

Richini-Pereira, V. B., Bosco, S. de M. G., Griese, J., Theodoro, R. C., Macoris, S. A. G., da Silva, R. J., \& Bagagli, E. (2008). Molecular detection of Paracoccidioides brasiliensis in road-killed wild animals. Medical Mycology, 46(1), 35-40. 10.1080/13693780701553002

Shikanai-Yasuda, M. A., Mendes, R. P., Colombo, A. L., Queiroz-Telles, F. de, Kono, A. S. G., Paniago, A. M. M., \& Martinez, R. (2017). Brazilian guidelines for the clinical management of paracoccidioidomycosis. Revista Da Sociedade Brasileira de Medicina Tropical, 50, 715-740. 10.1590/0037-86820230-2017

Shikanai-Yasuda, M. A., Telles Filho, F. de Q., Mendes, R. P., Colombo, A. L., \& Moretti, M. L. (2006). Consenso em paracoccidioidomicose. Revista da Sociedade Brasileira de Medicina Tropical, 39(3), 297-310. 10.1590/S0037-86822006000300017

Simões, L. B., Marques, S. A., \& Bagagli, E. (2004). Distribution of paracoccidioidomycosis: Determination of ecologic correlates through spatial analyses. Medical Mycology, 42(6), 517-523. 10.1080/13693780310001656795

Teixeira, M. de M., Theodoro, R. C., Oliveira, F. F. M. de, Machado, G. C., Hahn, R. C., Bagagli, E., \& Soares Felipe, M. S. (2014). Paracoccidioides lutzii sp. Nov.: Biological and clinical implications. Medical Mycology, 52(1), 19-28. 10.3109/13693786.2013.794311

Torrado, E., Castañeda, E., Hoz, F. de la, \& Restrepo, A. (2000). Definition of paracoccidioidomycosis endemic areas in Colombia. Biomédica, 20(4), 327334. 10.7705/biomedica.v20i4.1076

Toyotani, B. J. S., \& Souza, E. B. A. de. (2019). Incidência Da Paracoccidioidomicose No Estado De Rondônia - 2011 A 2014. Revista Saber Científico, 8(2), 104-113. 10.22614/resc-v8-n2-995 
Research, Society and Development, v. 10, n. 9, e31810918182, 2021

(CC BY 4.0) | ISSN 2525-3409 | DOI: http://dx.doi.org/10.33448/rsd-v10i9.18182

Trejo-Chávez, A., Ramírez-Romero, R., Ancer-Rodríguez, J., Nevárez-Garza, A. M., \& Rodríguez-Tovar, L. E. (2011). Disseminated paracoccidioidomycosis in a Southern two-toed sloth (Choloepus didactylus). Journal of Comparative Pathology, 144(2-3), 231-234. 10.1016/j.jcpa.2010.08.012

Vieira, G. de D., Alves, T. da C., Lima, S. M. D. de, Camargo, L. M. A., \& Sousa, C. M. de. (2014). Paracoccidioidomycosis in a western Brazilian Amazon State: Clinical-epidemiologic profile and spatial distribution of the disease. Revista Da Sociedade Brasileira de Medicina Tropical, 47, 63-68. 10.1590/00378682-0225-2013 